\title{
Der künstlerisch-ästhetische Aspekt im dramapädagogischen Fremdsprachenlernen
}

\author{
Ein Kurskonzept
}

\section{Alexandra Hensel}

\begin{abstract}
Ich möchte Ihnen im Folgenden einen Einblick in meine Theaterarbeit in einem DaF-Oberstufenkurs (C1) an der Universität Göttingen geben, der sich jeweils über ein Semester erstreckt. In diesem Kurs arbeiten wir mit einem sprachlernorientierten Schwerpunkt, verbunden mit einer künstlerisch-ästhetischen Zielsetzung. Das heißt, es geht neben der sprachlichen Entwicklung immer auch um das ästhetische Wirken von Kurzszenen und es gibt eine Abschlusspräsentation. Während des Kurses werden in Kleingruppen Kurzszenen zu verschiedenen Texten erarbeitet, gegenseitig präsentiert und reflektiert. In der Probenzeit erfolgt ein sehr intensives und wiederholendes Arbeiten an den Szenen, welches schließlich in die Abschlusspräsentation mündet. Meine These dabei ist, dass die Spracharbeit durch den künstlerisch-ästhetischen Aspekt nachhaltig gefördert wird, denn das wiederholende, variierende und öffentliche Arbeiten an den Szenen und das Präsentieren unterstützen sowohl die künstlerische als auch die sprachliche Entwicklung. Bereits während des Kurses und insbesondere gegen Ende des Kurses sind die Teilnehmer für die Sprache höchst motiviert. Sie haben sowohl ein detaillierteres Verständnis der Sprache und ihrer Gesten erlangt als auch ein vertieftes Sprachgefühl entwickelt. Dies führt zu mehr Selbstbewusstsein im Gebrauch der Sprache, was das spontane Sprechen im Alltag nachhaltig unterstützt. ${ }^{1}$
\end{abstract}

\section{Zur Kursstruktur und den Übungen}

Folgende Rahmenbedingungen bestehen: Der Kurs findet an der Universität einmal wöchentlich für 120 Minuten statt, die Teilnehmer sind internationale Studierende mit den Niveaus B2/C1 und in der Regel sind sie zwischen 19 und 25 Jahren, vereinzelt gibt es aber auch ältere Teilnehmer. Der Kurs setzt sich meistens aus ca. 12 Studierenden zusammen.

\footnotetext{
${ }^{1}$ Um den Lesefluss nicht zu sehr zu beeinträchtigen, besonders bei den Übungsbeschreibungen, benutze ich eine einheitliche Form, die die weibliche und die männliche Form einschließt.
} 
In der ersten Sitzung tauschen wir uns über unsere Theatererfahrungen und die Erwartungen an den Kurs aus. Anschließend erkläre ich den Ablauf des Kurses und empfehle, dass sich jeder hier ausprobieren - vielleicht auch einmal über seine Grenzen hinausschnuppern - sollte, aber natürlich jederzeit auch die Möglichkeit hat, etwas abzulehnen, was ihm nicht zusagt. Das halte ich besonders in einem internationalen Kurs für wichtig, denn Theaterarbeit ist auch körperlich. Fast jeder präsentiert sich zum ersten Mal und aus der jeweils eigenen Perspektive gibt es diesbezüglich eine andere Wahrnehmung. Dies soll auch im Kurs bewusst gemacht werden. Jeder sollte sich ebenfalls dazu verpflichten, regelmäßig und pünktlich zu erscheinen, da es die ganze Theatergruppe betrifft, wenn ein Teilnehmer fehlt. In aller Regel gelingt dies auch.

Der Ablauf für die kommenden Stunden ist folgender:

- Warm-up

- Text-/Szenenerarbeitungen

- Abschluss

\subsection{Warm-up}

Beim Warm-up machen wir beispielsweise folgende Übungen:

- Körper abklopfen: Einer beugt sich nach vorn, Knie und Kopf locker, der andere klopft den Rücken, die Arme und die Beine ab. Danach wird alles ausgestrichen und mit zwei Fingern läuft der eine Partner die Wirbelsäule des anderen hoch, der sich dementsprechend langsam hochrollt.

- Ja-Nein-Doch-Kreis: Mit einem Klatsch wird ein lautes JA an den Nachbarn weitergegeben, mit einem NEIN und einer Stopphaltung mit der Hand wird die Richtung gewechselt, und mit einem DOCH kann man nach einem NEIN einer gegenüber stehenden Person den Impuls schicken. Das Ganze funktioniert auch mit anderen Vokabeln.

- Fangen spielen: Einer ist der Fänger und muss einen anderen antippen. Dieser kann sich dadurch retten, dass er den Namen eines anderen Mitspielers ruft.

- Zickzack-Dialog: Es wird ein Thema vorgegeben wie "Streitgespräch", dazu sollen sich die Teilnehmer zu zweit einen Anfangsdialog überlegen. Nach ein paar Minuten stellen sich alle in zwei Reihen auf, die Partner stehen dabei jeweils gegenüber. Das erste Paar beginnt, und im Zickzack müssen alle spontan auf die vorherige Äußerung reagieren.

- Zwei-Stühle-Improvisation: Zwei Stühle stehen im Raum und sollen spontan bespielt werden: Wo stehen diese Stühle? Es können Szenen beim Arzt, im Kino, auf einer Parkbank etc. entstehen. 


\subsection{Text- und Szenenerarbeitung}

In dieser Phase gibt es in der Regel jedes Mal einen neuen Kurztext, zum Beispiel Herzstück (Heiner Müller) oder Zweikampf I (Fitzgerald Kusz) sowie Gedichte wie Sachliche Romanze (Erich Kästner), Sketche (Loriot) oder Märchen. Selbst die Gretchen-Szene aus Goethes Faust eignet sich.

Die Teilnehmer sollen jedes Mal in Kleingruppen von zwei bis vier Teilnehmern kleine Szenen zum Text erarbeiten, dies immer vor dem Hintergrund: Wer sind die Personen? Welche Beziehung haben sie zueinander? Warum sagen sie das? Wie kann es weitergehen? Dazu kann ein Arbeitsblatt ausgegeben werden. Nach ca. zehn Minuten werden die verschiedenen Szenen gegenseitig vorgespielt. Im Anschluss hieran sollen die Zuschauer beschreiben, was sie gesehen haben, wie es gewirkt hat und was man eventuell größer oder deutlicher machen könnte. Als Übungsleiterin moderiere ich hier und gebe auch ein Feedback. Meistens kann die Szene dann mit den Veränderungen noch ein zweites Mal gespielt werden.

Um einen detaillierteren Einblick zu bekommen, möchte ich die Erarbeitung der Gretchen-Szene beschreiben: Zunächst hatten zwei Gruppen jeweils eine Geräuschkulisse erarbeitet, d.h. zu einer Landschaft wie Wald oder Strand sollte sich jede Gruppe die Geräusche überlegen und diese der anderen Gruppe, die mit geschlossenen Augen auf dem Boden saß, vorführen. Dann habe ich zwei Sätze vorgegeben:

1. Wartest du schon lange?

2. Ja, eine halbe Ewigkeit.

Jede Gruppe sollte nun eine kleine Szene mit diesen Sätzen und der Geräuschkulisse erarbeiten. Im Anschluss hieran machte ich die Teilnehmer mit dem Element des Gedankensprechens vertraut: Eine weitere Person steht hinter der eigentlichen Figur und spricht deren Gedanken laut aus. Danach gab ich ihnen die Begegnungsszene von Faust und Margarete, die wir im Kreis gemeinsam laut lasen, jeder eine Zeile:

Faust: Mein schönes Fräulein, darf ich wagen,

Meinen Arm und Geleit Ihr anzutragen?

Margarete: Bin weder Fräulein, weder schön,

Kann ungeleitet nach Hause gehn.

(Goethes Faust, 1899, S. 128.)

Nachdem Vokabelfragen geklärt wurden, sollte diese Szene zunächst klassisch dargestellt werden. Danach sollte sie in der einen Gruppe ins Jetzt übertragen und von der anderen Gruppe sehr modern interpretiert werden. Es entstanden beeindruckende Szenen mit Hintergrund bzw. Ambiente (im Park und in der Disco) und klar definierten Charakteren. Bei der Präsentation, Betrachtung und Reflexion der Kurzszenen wird also sowohl das künstlerische Auge als auch die Spracharbeit gefördert. 


\section{Probenzeit und Werkschau}

In den letzten vier Wochen beginnt die Probenzeit, die in die Abschlusspräsentation mündet. Ich nenne sie Werkschau, da gezeigt wird, was "gewerkelt" wurde und der Anspruch einer kompletten Aufführung gemildert wird. Die Teilnehmer teilen zunächst in einem Brainstorm-Verfahren mit, welche Übungen und Szenen ihnen am besten gefallen haben. Sie sollen motiviert sein, diese zeigen zu wollen. Dann erstelle ich zum nächsten Treffen ein Grundgerüst, wie die Werkschau aufgebaut sein könnte, und präsentiere dieses Gerüst den Teilnehmern. Sie müssen nun dieses Gerüst füllen. Anfangs herrscht Verwirrung, da vieles noch offen ist und die genauen Dialoge noch festgehalten werden müssen. Doch nach und nach finden sich alle in die Arbeit ein. Die Teilnehmer beginnen, immer reger miteinander zu diskutieren: Wie, wo und mit wem kann etwas dargestellt werden? Wichtig hierbei ist, den Teilnehmern immer mehr Freiheit und Eigenständigkeit zuzugestehen, denn es soll ihre Werkschau werden. Die hohe Identifikation mit der Werkschau führt zu noch mehr Einsatz und Motivation. Dazu gehört auch, dass sie ihre Texte lernen und sich auch einmal privat treffen, um gemeinsam eine Szene zu üben. Szenen werden in der Probenzeit mehrfach hintereinander einstudiert, sodass auch hier die Sprache im künstlerischen Rahmen geübt und verfeinert wird. Als Übungsleiterin achte ich in dieser Phase auf den Ablauf und den sprachlichen Ausdruck.

Dadurch, dass die Werkschau am Ende stattfindet - besonders in den letzten Wochen wird intensiv auf dieses Ziel hingearbeitet -, herrscht ein hoher Anreiz, gut sein zu wollen. Die Werkschau fordert zudem den "Gang durchs Nadelöhr", denn eine der größten Hürden beim Lernen fremder Sprachen ist es, vor anderen und mit anderen zu sprechen. Dieser "Gang durchs Nadelöhr" wird am Ende durch den Applaus und das Gefühl, es geschafft zu haben, belohnt. Diese Freude kann kaum größer sein! Die Teilnehmer wünschen sich meistens, spontaner sprechen zu können. Dies wird mit dieser Arbeit intensiv geübt. Sie erlangen mehr Selbstbewusstsein im Gebrauch der Fremdsprache. Durch die Ganzheitlichkeit des Unterrichts, die Theaterarbeit generell bietet, wird die Sprache detaillierter und im Kontext geübt. Auf diese Weise wird die Handlungsfähigkeit des Teilnehmers unterstützt. Hinzu kommt, dass die Ästhetik der Fremdsprache in einem künstlerischen Rahmen geschult wird. Außerdem sind die Teilnehmer interkulturell kompetenter geworden, da die Durchführung eines gemeinsamen Projektes mitverschiedenen Persönlichkeiten und kulturellen Hintergründen viel Sensibilität und Empathie erfordert. Es entsteht dabei auch ein positives Gruppengefühl - ich lerne nicht allein -, was letztlich wiederum den Lernprozess unterstützt.

\section{Die Rolle des Übungsleiters}

Als Übungsleiter sollte man eine offene, sensible und moderierende Haltung einnehmen. Es geht darum, die Teilnehmer mit Fragen und positivem Feedback zu motivieren. Darüber hinaus ist es notwendig, die Arbeit kritisch zu beleuchten. 
Die Studierenden sollten dazu befähigt werden, eigenständig und reflektiert in einen Austausch zu treten. Für besonders wichtig halte ich es, dass man als Übungsleiter selbst Theater gespielt haben sollte. Denn andernfalls kann man die Hürden, die die Teilnehmer überwinden, nicht nachempfinden und es kommt schnell zur Überforderung. Dies hätte genau den gegenteiligen Effekt, nämlich Frustration.

\section{Fazit}

Wie an mehreren Stellen bereits erwähnt, bieten die Theaterarbeit im Allgemeinen und der ästhetisch-künstlerische Aspekt im Fremdsprachenunterricht im Speziellen viele Vorteile für den Teilnehmer, sich sprachlich und persönlich weiterzuentwickeln. Die Fremdsprache wird in ihrer Komplexität gelernt - mit Gesten, Ausdruck, Stimmlage, Mimik. Durch die ästhetisch-künstlerische Komponente wird die Fremdsprache erspürt, erfahren und differenziert beleuchtet. Außerdem werden verschiedene Texte erarbeitet, die Bestandteil der deutschen Literatur- und Kulturgeschichte sind. All dies bietet letztlich die Möglichkeit, sowohl zukünftige Gespräche und Begegnungen als auch den Unterrichtsalltag auf bewusstere, intensivere und vielseitigere Weise zu gestalten. 